\title{
Monopoles and Their Spectral Data
}

\author{
Jacques Hurtubise $^{1 \star}$ and Michael K. Murray ${ }^{2}$ \\ ${ }^{1}$ Department of Mathematics, McGill University, Burnside Hall, 805 Sherbrooke St. W., Montréal, \\ Québec, Canada, H3A 2K6 \\ ${ }^{2}$ Department of Mathematics, R.S.Phys.S., The Australian National University, GPO Box 4, Canberra, \\ ACT 2601, Australia
}

Received July 11, 1989; in revised form February 12, 1990

\begin{abstract}
A new definition of spectral data of a monopole is given for any compact Lie or Kac-Moody group. It is shown that the spectral data determines the irreducible monopole. In the case of maximal symmetry breaking the spectral data is shown to reduce to an earlier definition in terms of algebraic curves indexed by the nodes of the Dynkin diagram of the group. The structure of solutions to Nahm's equations corresponding to the monopole is discussed.
\end{abstract}

\section{Introduction}

In 1982 Hitchin defined the spectral curve of an $S U(2)$ monopole which is the collection of lines in $\mathbf{R}^{3}$ along which a certain differential operator has non-zero $\mathscr{L}^{2}$ kernel. These lines form an algebraic curve in the minitwistor space $\mathscr{T}=T\left(P_{1}(\mathbf{C})\right)$ of all oriented lines in $\mathbf{R}^{3}$. The monopole is equivalent, via the twistor correspondence, to a certain holomorphic bundle on $\mathscr{T}$ and Hitchin showed that this bundle and therefore the monopole was determined by the spectral curve.

For other compact groups the definition of the spectral curve was generalised to the spectral data of a monopole in Murray (1984). The spectral data is a collection of curves $S_{i}$, indexed by the nodes of the Dynkin diagram of the group, and a division of the intersections $S_{i} \cap S_{j}$ into two pieces $S_{i, j}$ and $S_{j, i}$ when the nodes $i$ and $j$ are joined on the Dynkin diagram. For the groups $S U(n), S p(n)$ and $S O(2 n)$ it was shown in Murray (1984) that a general monopole (one for which the curves are reduced and for which their intersections are distinct points) is determined by its spectral data. Most recently in Hurtubise and Murray (1989) the present authors showed, amongst other things, that the same result was true for $S O(2 n+1)$.

The methods used to prove these results all relied in an essential way on the linearity inherent in the vector bundles involved rather than on the group structure of the principal bundles. This is why the proofs were difficult to extend beyond the classical groups. The original definition of the spectral data in Murray (1984)

\footnotetext{
$\star$ Research supported in part by NSER C grant A8361 and FCAR grant EQ3518
} 
however used principal bundles and the structure of the Bruhat cells on $G / \bar{B}$ and did not distinguish between the classical and exceptional groups. We give below a proof that the spectral data determines a general, irreducible monopole for any compact group or Kac-Moody group using a method that shows that the spectral data determines the transition function of the holomorphic principal bundle which is equivalent to the monopole. This is a quite different method from those used previously and gives a more complete result.

This approach has motivated us to revise the definition of the spectral data of the monopole. We shall define the spectral data to be a curve in $\mathscr{T}$ and a section of a certain sheaf (determined by the asymptotics of the monopole) supported over this curve. This new definition is somewhat more abstract than what we had before however it has two advantages. Firstly it is easy to see that for any type of symmetry breaking the monopole is always determined by its spectral data without any assumption of genericity. Secondly for generic, maximal symmetry breaking we can show that this definition is equivalent to the older one mentioned above. This proves that the spectral curves and the division of their intersections determines the general monopole.

Before we begin let us make some notational comments about principal bundles and also outline the groups involved. Let $Q(\mathscr{T}, G)$ be a principal bundle over $\mathscr{T}$ with total space $Q$ and group $G$. Then $G$ acts transitively and freely on the fibres of the projection $Q \rightarrow \mathscr{T}$. If $Y$ is any set on which $G$ acts on the left we can form the fibration $Q \times{ }_{G} Y$ which is the orbit space of the right $G$ action $(q, y) g=\left(q g, g^{-1} y\right)$ on the product $Q \times Y$. We denote the orbit of $(q, y)$ by $[q, y]_{G}$ and use the notation $Q(Y)$ for $Q \times{ }_{G} Y$. The general philosophy of this construction is that the fibres of $Q(H) \rightarrow \mathscr{T}$ have "whatever structure" $Y$ has that is preserved by group action. As a number of cases will be important below let us list them here.

1.1. When $Y$ is a vector space and $G$ acts by a representation $\lambda, Q(Y)$ is a vector bundle and we shall in addition employ the notation $Q(\lambda)$ for the vector bundle $Q(Y)$.

1.2. If $G$ acts by group automorphisms on another group $H$ then $Q(H)$ is a bundle of groups. That is each fibre of the projection $Q(H) \rightarrow \mathscr{T}$ is a group with the product $[q, h]_{G}\left[q, h^{\prime}\right]_{G}=\left[q, h h^{\prime}\right]_{G}$.

1.3. If $G$ acts on the left of a group $H$ and the action commutes with the right action of $H$ on itself then $Q(H)$ is a principal $H$ bundle with the action $[q, h]_{G} h^{\prime}=\left[q, h h^{\prime}\right]_{G}$. A trivial example is that $Q(G)$ is the bundle $Q$ itself under the isomorphism $[q, g]_{G} \mapsto q g$.

1.4. Another example of 1.3 is if we are given a homomorphism $\chi: G \rightarrow H$. Then there is an $H$ bundle induced, most simply, by composing the transition functions of $Q$ with $\chi$. More invariantly let $G$ act on $H$ by $g \cdot h=\chi(g) h$, then $Q(H)$ is the induced $H$ bundle.

Recall that a subset $R$ of a principal $G$ bundle $Q$ is called a reduction of $Q$ to a subgroup $B \subset G$ if it is stable under $B$ and with respect to the action of $B$ is a principal $B$ bundle. In such a case if $G$ acts on $Y$ and $B$ acts on $Y$ by virtue of being a subgroup of $G$ then there is a canonical identification of the two associated 
fibrations

$$
\begin{gathered}
R(Y)=Q(Y), \\
{[r, y]_{B} \mapsto[r, y]_{G} .}
\end{gathered}
$$

The notation for the groups we shall be using is:

$K$ a compact Lie group or a Kac-Moody group, $C\left(\Phi^{\infty}(\star)\right)$ the centraliser in $K$ of an element $\Phi^{\infty}(\star)$ of the Lie algebra $L K$ of $K$, $G$ the complexification of $K$,

$T$ a maximal torus of $K$ contained in $C\left(\Phi^{\infty}(\star)\right)$,

$T^{\mathbf{c}}$ the complexification of $T$,

$P$ a parabolic subgroup of $G$,

$\bar{P}$ an opposite parabolic,

$L=P \cap \bar{P}$ the complexification of $C\left(\Phi^{\infty}(\star)\right)$ and

$V$ and $\bar{V}$ which are the unipotent radicals of $P$ and $\bar{P}$ respectively.

The organization of the paper is as follows. In Sect. 2, we recall how a monopole defines a principal $G$-bundle $Q$ over $\mathscr{T}$, as well as reductions, $R^{+}, R^{-}$or $Q$ to $P, \bar{P}$ respectively. By the exact sequence of groups

$$
0 \rightarrow V \rightarrow P \rightarrow L \rightarrow 0
$$

and the induction mentioned above (1.4) $R^{+}$defines an $L$ bundle $M$. We show that the isomorphism class of $M$ belongs to a discrete set determined by the asymptotics of the monopole; it should be thought of as fixed.

In Sect. 3, we show how to classify the $P$-bundles with induced $L$ bundle isomorphic to $M$. In a generalisation of the construction of a principal bundle from transition functions these bundles are shown to be determined by "transition sections" taking values in a bundle of groups. The spectral data is then defined: the reduction $R^{-}$defines a section of $R^{+}(G / \bar{P})$ whose "principal part" is the spectral data. The notion of principal part of a map into $G / \bar{P}$ was introduced by Gravesen and Segal (Gravesen (1987)) and generalises the classical principal part of a meromorphic map (see Sect. 3(ii) for Gravesen's definition). In our case, this idea is extended to a bundle context; the principal part of $R^{-}$is supported on a curve in $\mathscr{T}$, the spectral curve. The spectral curve and the restriction of the principal part to the curve are the spectral data of the monopole. We show that they determine the monopole.

Section 4 is devoted to showing that the spectral data in the case of general monopoles with maximal symmetry breaking reduces to the previous definition of Murray (1984), that is, a collection of curves indexed by the nodes of the Dynkin diagram of the group and a partition of the intersection of any two curves joined on the Dynkin diagram into two equal sets of points. This is used to show that such curves and points determine the general, maximal symmetry breaking monopole for any group $G$.

In Sect. 5, we discuss solutions to Nahm's equations. For the classical groups, in the case of maximal symmetry breaking these were shown in Hurtubise and Murray (1989) to encode the structure of the monopole. In this more general case, 
when $K$ is a compact group and for arbitrary symmetry breaking one can define a solution to Nahm's equations associated to any representation of $K$. We make some comments on the structure of these solutions.

\section{Monopoles}

A monopole for a Kac-Moody or compact Lie group $K$ with Lie algebra $L K$ is a pair $(A, \Phi)$ such that

i) $A$ is an $L K$ valued 1 -form (connection) on $\mathbf{R}^{3}$ and $\Phi$ is an $L K$ valued function (called the Higgs field),

ii) $(A, \Phi)$ satisfies the Bogomolny equations

iii) and the action

$$
* d_{A} \Phi=F_{A},
$$

$$
\int_{\mathbf{R}^{3}}\left|F_{A}\right|^{2}+\left|d_{A} \Phi\right|^{2}
$$

is finite.

When $K$ is a Kac-Moody group these monopoles are also called calorons, see Nahm (1983) and Garland and Murray (1989)

As a consequence of the finiteness of the action the monopole satisfies the Bogomolny, Prasad, Sommerfield (BPS) boundary conditions, in particular the Higgs field has a limit over $S_{\infty}^{2}$, the sphere at infinity in $\mathbf{R}^{3}$ :

$$
\Phi^{\infty}: S_{\infty}^{2} \rightarrow L K
$$

whose image lies on an adjoint orbit of the group.

Fixing a point $\star$ in $S_{\infty}^{2}$, we are interested in the set of all monopoles $(A, \Phi)$, where the Higgs field has a limit at infinity and $\Phi^{\infty}(\star)$ is fixed; modulo the action of the group of gauge transformations with limits at infinity such that $g^{\infty}(\star)=1$. The resulting moduli space is the moduli space of based monopoles. To remove the basepointing condition we would have to allow gauge transformations with $g^{\infty}(\star)$ taking values in the subgroup $C\left(\Phi^{\infty}(\star)\right)$ of $K$ consisting of elements which leave $\Phi^{\infty}(\star)$ fixed. The moduli space of based irreducible monopoles fibres over the moduli space of unbased irreducible monopoles with fibre $C\left(\Phi^{\infty}(\star)\right)$.

With these definitions we have an explicit realisation of the adjoint orbit and

$$
\Phi^{\infty}: S_{\infty}^{2} \rightarrow K / C\left(\Phi^{\infty}(\star)\right)
$$

The Higgs field at infinity defines an element of the second homotopy group of $K / C\left(\Phi^{\infty}(\star)\right)$ which is isomorphic to $\mathrm{Z}^{r-d}$, where $r-d$ is the dimension of the torus generated by $\Phi^{\infty}(\star)$. To find an explicit set of generators for this homotopy group we proceed as follows.

We choose a maximal torus $T$, whose Lie algebra contains $\Phi^{\infty}(\star)$, and a set of $r$ simple roots $\Delta$ and denote by $\Delta_{0}$ the set of $d$ simple roots vanishing on $\Phi^{\infty}(\star)$. The subgroup $W_{0}$ of the Weyl group $W$ generated by the simple root reflections for simple roots in $\Delta_{0}$ is the subgroup stabilizing $\Phi^{\infty}(\star)$. There is an identification

$$
K / C\left(\Phi^{\infty}(\star)\right) \cong G / \bar{P}
$$


where $\bar{P}$ is the parabolic subgroup containing the negative root spaces and the positive root spaces for roots in $\Delta_{0}$. The magnetic charges of the monopole are the collection of $r-d$ (non-negative) integers indexed by the elements of $\Delta$ not in $\Delta_{0}$ and defined by

$$
m_{i}=c_{1}\left(\Phi^{\infty *} \mathscr{L}\left(\lambda_{i}\right)\right)
$$

where $\lambda_{i}$ is a fundamental weight dual to a simple root not in $\Delta_{0}$ and $\mathscr{L}\left(\lambda_{i}\right)$ is the homogeneous line bundle over $G / \bar{P}$ obtained by extending $\lambda_{i}$ to a holomorphic character of $\bar{P}$.

The Higgs field at infinity determines a reduction of the (trivial) $K$ bundle at infinity to $C\left(\Phi^{\infty}((\star))\right.$. The connection has a limit at infinity (Hitchin (1982)) which satisfies $\nabla \Phi^{\infty}=0$ and therefore the connection reduces to the $C\left(\Phi^{\infty}(\star)\right)$ subbundle. This connection gives the complexification of the $C\left(\Phi^{\infty}(\star)\right)$ bundle a holomorphic structure. We shall denote this holomorphic $L$ bundle over $\mathbf{P}_{1}$, the sphere at infinity, by $W^{+}$. As the dual of the curvature also satisfies $\nabla * F^{\infty}=0$ we have a further holomorphic reduction to $C\left(* F^{\infty}(\star)\right)$. Finally by a theorem of Grothendieck (1975), even if $F^{\infty}(\star)$ is not regular, we can reduce this bundle to $T^{\mathbf{c}}$. This holomorphic bundle (which determines $W^{+}$by the embedding $T^{\mathfrak{c}} \rightarrow L$ ) is a sum of line bundles determined by their Chern classes which are in turn determined by $F^{\infty}(\star)$. Note that if $\Phi^{\infty}(\star)$ is not regular $* F^{\infty}(\star)$ contains more information than its magnetic charges. This information takes the form of integers which label strata inside the moduli space of monopoles. See Murray (1989) for more details when $K=S U(N)$. In any case, the possibilities for $W^{+}$are discrete. Similarly we define $W^{-}$which is the conjugate of the pull-back of $W^{+}$under the antipodal map.

By the twistor correspondence a monopole determines a holomorphic principal $G$ bundle $Q$, where $G$ is the complexification of $K$, over $\mathscr{T}$ the so-called minitwistor space of oriented lines in $\mathbf{R}^{3}$. As a complex manifold $\mathscr{T}$ is isomorphic to the tangent space of one dimensional complex projective space. For each oriented line $\gamma$ we define a $G$-space by

$$
Q_{\gamma}=\left\{g \in C^{\infty}(\gamma, G) \mid\left\langle g^{-1} A g, \frac{\partial}{\partial t}\right\rangle+g^{-1} \frac{\partial g}{\partial t}=i g^{-1} \Phi g\right\},
$$

where $g$ is a map from $\gamma$ to $G$ and $t$ is a normalised linear parameter for $\gamma$ in the direction of its orientation. The union $Q$ of these $G$-spaces forms a holomorphic principal $G$ bundle on $\mathscr{T}$. Letting $g$ be the exponential of a map $s$ from $\gamma$ to $L G$ we deduce that the fibre of the adjoint bundle ad $Q$ over $\gamma$ is

$$
\operatorname{ad} Q_{\gamma}=\left\{s \in C^{\infty}(\gamma, L G) \mid \nabla_{\gamma} s-[i \Phi, s]=0\right\} .
$$

The monopole can be reconstructed from this bundle; for details see Hitchin (1982) and Murray (1984) when the group is a compact Lie group and Garland and Murray (1989) when it is a Kac-Moody group.

If we define ad $R^{+}$to be the subbundle of $s$ in ad $Q_{\gamma}$ that are bounded as we approach $+\infty$ then this is a bundle of parabolic subalgebras whose fibres are isomorphic to $L P$. For details see Murray (1984). This determines a reduction of $Q$ to a $P$ bundle $R^{+}$whose adjoint bundle is this bundle of Lie algebras. In a similar fashion by looking at bounded solutions in the direction of $-\infty$ we obtain 
a reduction $R^{-}$to $\bar{P}$. When we project out the unipotent radical of the parabolics via the maps.

$$
V \rightarrow P \rightarrow L
$$

and

$$
\bar{V} \rightarrow \bar{P} \rightarrow L
$$

we obtain induced $L$ bundles $M^{+}=R^{+}(L)$ and $M^{-}=R^{-}(L)$.

By applying a generalisation of the methods in Hitchin (1982), pp. 590-594 we can show that

$$
\begin{aligned}
& M^{+}=\mathscr{L}^{\Phi \infty(\star)} \otimes \pi^{*} W^{+}, \\
& M^{-}=\mathscr{L}^{\Phi \infty(\star)} \otimes \pi^{*} W^{-},
\end{aligned}
$$

where $W^{+}$and $W^{-}$are the bundles at infinity defined above, $\pi: \mathscr{T} \rightarrow \mathbf{P}_{1}$ is the projection and the bundle $\mathscr{L}^{\Phi \infty(\star)}$, as well as the "tensor product" are defined as follows. Let $(\zeta, \eta) \mapsto \eta(d / d \zeta)$ be the standard co-ordinates on $\mathscr{T}$ and $U_{0}=\{\eta \neq \infty\}$, $U_{1}=\{\eta \neq 0\}$ the standard cover by two open sets. Then $\mathscr{L}^{\Phi \infty(\star)}$ is the $T^{\mathrm{C}}$ bundle with transition function

$$
f_{01}=\exp \left(-i \frac{\eta}{\zeta} \Phi^{\infty}(\star)\right)
$$

from $U_{0}$ to $U_{1}$. To put it more concisely $\mathscr{L}^{\Phi(\star)}$ is the twistor bundle for a $T$ monopole with zero connection and constant Higgs field equal to $\Phi^{\infty}(\star)$. The tensor product in (2.11) is defined by multiplying the transition functions of the respective bundles. This makes sense (that is the cocycle condition is satisfied for the product) because $L$ is the centralizer of $\Phi^{\infty}(\star)$.

The justification for the use of the tensor product notation is that if $\chi$ is an irreducible representation of $L$ (with highest weight also denoted $\chi$ ) then one has a line bundle $\mathscr{L}^{\chi\left(\Phi^{\infty}(*)\right)}$ corresponding to the weight $\chi$ and

$$
\begin{aligned}
& M^{+}(\chi)=\mathscr{L}^{\chi\left(\Phi^{\infty}\right)(*)} \otimes \pi^{*} W^{+}(\chi), \\
& M^{-}(\chi)=\mathscr{L}^{\chi\left(\Phi^{\infty}\right)(\star)} \otimes \pi^{*} W^{-}(\chi) .
\end{aligned}
$$

As these are framed monopoles we have an actual identification of the bundles in (2.11) over the fibre of $\pi$ consisting of all lines that point in the direction $\star$ and of the bundles in (2.12) over the fibre of lines that point in the opposite direction to $\star$. In these case of maximal symmetry breaking, when $L$ is abelian this defines a unique identification of the bundles in (2.11) and (2.12) over the whole of $\mathscr{T}$.

We have now shown that $M^{+}$belongs to a discrete set and is determined by the boundary conditions of the monopole. We now fix $\mathrm{M}^{+}$and ask what extra information determines the monopole.

\section{The Spectral Data}

Reductions, such as $R^{-}$, of the holomorphic principal bundle $Q$ to a parabolic subgroup $\bar{P}$ are in bijective correspondence with the holomorphic sections of the 
fibration $Q(G / \bar{P})$ (see for instance Koboyashi and Nomizu (1989).) Because $R^{+}$is also a reduction of $Q$ recall from (1.5) that we have a natural isomorphism

$$
R^{+}(G / \bar{P})=Q(G / \bar{P})
$$

and $R^{-}$therefore determines a section of $R^{+}(G / \bar{P})$. Before exploiting this fact to define the spectral data, we will first classify the $P$ bundles, such as $R^{+}$, whose induced $L$ bundles is isomorphic to $M$ (see (1.4)). More precisely we will classify pairs consisting of a $P$ bundle $R^{+}$whose induced $L$ bundle, $R^{+}(L)$ is isomorphic to $M$ and a choice of such an isomorphism. The different isomorphisms are all obtained by acting by the automorphism group of $M$ and we will consider the effect of this later. Because there is the projection $R^{+} \rightarrow R^{+}(L), r \mapsto[r, 1]_{L}$, choosing the isomorphism defines a map $R^{+} \rightarrow M$ which commutes with the action of $P$, where $P$ acts on the second bundle via the homomorphism $P \rightarrow L$ (see also (1.4)). Hence we call such bundles " $P$-bundles projecting to $M$."

i) P-Bundles Projecting to $M$. Let us fix a trivialisation, or a collection of local sections $\left\{\hat{\phi}_{\alpha}\right\}$ of $M$ with respect to an open cover $\left\{U_{\alpha}\right\}$ of $\mathscr{T}$. Because all holomorphic bundles are trivial over a Stein open set we can fix a Stein open cover. Then there are transition functions

$$
l_{\alpha \beta}: U_{\alpha} \cap U_{\beta} \rightarrow L
$$

defined by

$$
\hat{\phi}_{\beta}=\hat{\phi}_{\alpha} l_{\alpha \beta} .
$$

Choose local sections $\phi_{\alpha}$ of $R^{+}$which project to the local sections $\hat{\phi}_{\alpha}$ of $M$. Then the bundle $R^{+}$and therefore $Q=R^{+}(G)$, and hence the monopole, is determined by the transition functions

$$
p_{\alpha \beta}: U_{\alpha} \cap U_{\beta} \rightarrow P
$$

which satisfy

$$
\phi_{\beta}=\phi_{\alpha} p_{\alpha \beta} .
$$

Because the group $P$ is a semidirect product of $V$ and $L$ we can write $p_{\alpha \beta}=l_{\alpha \beta} v_{\alpha \beta}$. The transition functions $l_{\alpha \beta}$ are determined by our fixing of the bundle $M$ and its local trivialisation. To determine the bundle $Q$ we need only recover the $v_{\alpha \beta}$, but what sort of objects are they? Some manipulating of the cocycle identities

$$
p_{\alpha \gamma}=p_{\alpha \beta} p_{\beta \gamma}
$$

and

$$
l_{\alpha \gamma}=l_{\alpha \beta} l_{\beta \gamma}
$$

will convince the reader that the $v_{\alpha \beta}$ are not themselves transition functions. We will show however, that correctly interpreted, they are transition sections.

Over the set $U_{\alpha}$ the trivialisations define an isomorphism

$$
\chi_{\alpha}:\left.M(P) \cong R^{+}\right|_{U_{\alpha}}, \quad\left[\hat{\phi}_{\alpha}, p\right]_{L} \mapsto \phi_{\alpha} p .
$$

The bundle $R^{+}$can therefore be thought of as made up of copies of the given 
$P$ bundle $M(P)$ over each of the sets $U_{\alpha}$ glued together by the bundle automorphisms $\chi_{\alpha}^{-1} \chi_{\beta}$ on the overlaps $U_{\alpha} \cap U_{\beta}$. This is just the same as the way a bundle with transition functions $g_{\alpha \beta}$ is thought of as copies of the trivial bundle over each $U_{\alpha}$ glued together with the transition functions on the overlaps.

We can calculate the maps $\chi_{\alpha}^{-1} \chi_{\beta}$ from the explicit form (3.4). A typical element $\left[\hat{\phi}_{\beta}, p\right]_{L}$ in $M(P)$ is mapped by $\chi_{\beta}$ to $\phi_{\beta} p$ in $R^{+}$. This is the same as $\phi_{\alpha} p_{\alpha \beta} p$ from (3.3) and therefore under $\chi_{\alpha}$ must have come from

$$
\begin{aligned}
{\left[\hat{\phi}_{\alpha}, p_{\alpha \beta} p\right]_{L} } & =\left[\hat{\phi}_{\alpha}, l_{\alpha \beta} v_{\alpha \beta} p\right]_{L} \\
& =\left[\hat{\phi}_{\alpha} l_{\alpha \beta}, v_{\alpha \beta} p\right]_{L} \\
& =\left[\hat{\phi}_{\beta}, v_{\alpha \beta} p\right]_{L} \\
& =\left[\hat{\phi}_{\beta}, v_{\alpha \beta}\right]_{L}\left[\hat{\phi}_{\beta}, p\right]_{L} .
\end{aligned}
$$

where the last line represents the action of an element of the associated bundle of groups $M(V)$ (where $L$ acts on $V$ by conjugation; recall that $L$ is also a subgroup of $P$ and normalises $V$ ) on the associated fibration $M(P)$. It can be seen that this action commutes with the action of $P$ on $M(P)$; that is, it is an action by bundle automorphisms. If we define sections of the bundle of groups $M(V)$ over $U_{\alpha} \cup U_{\beta}$ by

$$
\hat{v}_{\alpha \beta}=\left[\hat{\phi}_{\beta}, v_{\alpha \beta}\right]_{L}
$$

then these transition sections define the bundle $R^{+}$. It can be easily checked that these transition sections satisfy a co-cycle identity and that if we change to a new trivialisation $\phi_{\alpha}^{\prime}$ with $\phi_{\alpha}^{\prime}=\phi_{\alpha} v_{\alpha}$ then we have

$$
v_{\alpha \beta}^{\prime}=\left[\hat{\phi}_{\alpha}, v_{\alpha}^{-1}\right]_{L} v_{\alpha \beta}\left[\hat{\phi}_{\beta}, v_{\beta}\right]_{L} .
$$

Notice that this is all the freedom we have in changing the local sections $\phi_{\alpha}$ as we require them to project to the given sections $\hat{\phi}_{\alpha}$ of $M$.

We see now that the bundle $R^{+}$can be defined using these transition sections and that they behave just like the more familiar transition functions. When considering transition functions $g_{\alpha \beta}$ for a $G$ bundle $P \rightarrow X$ it is sometimes useful to introduce non-abelian sheaf cohomology. The set of all $G$ bundles is then in bijective correspondence with the sheaf cohomology set $H^{1}(X, G)$. In this setting the transition functions define representatives for the cohomology class corresponding to the bundle $P$. In our case a similar theory can be defined and the set of all bundles $R^{+}$with isomorphisms $R^{+}(L) \cong M$ shown to be equivalent to the set $H^{1}(\mathscr{T}, M(V))$. However we will not need this machinery.

ii) The Definition of the Spectral Data. The group $P$ acts on $G / \bar{P}$ and its action on the coset $\bar{P}$ sweeps out a dense open set. The subgroup $V$ acts freely and transitively on this orbit so it is isomorphic to $V$ itself. The complement of this set is an algebraic variety about which we shall say more later. This structure theory is well known for the case of Lie groups, see for instance Humphreys (1981) and for the case of Kac-Moody groups see Pressley and Segal (1986). We shall identify $V$ with this orbit $V \bar{P}$ and regard $G / \bar{P}$ as a "compactification" of $V$. We shall call the sheaf of holomorphic maps into $G / \bar{P}$ which intersect $V \bar{P}$ the sheaf of meromorphic maps and denote it by $\mathscr{M r}$ (by requiring that the image of the map 
intersect $V \bar{P}$ we are excluding maps that are "infinite" everywhere). The sheaf $\mathcal{O}(V)$ of maps into the group $V$ acts naturally on $\mathscr{M r}$, and Gravesen (1987) defines the quotient sheaf $\mathscr{P} r=\mathscr{M} r / \mathcal{O}(V)$ to be the sheaf of principal parts. This generalises the classical case where $G / \bar{P}$ is one dimensional complex projective space, $V \bar{P}$ is $\mathbf{C}$ and the complement is the "point at infinity."

Now we return to the section of $R^{+}(G / \bar{P})$ defined by the reduction $R^{-}$and mentioned at the beginning of Sect. 3. Locally this section takes the form $\left[\phi_{\alpha}, f_{\alpha}\right]_{p}$, where $f_{\alpha}: U_{\alpha} \rightarrow G / \bar{P}$ are defined by

$$
R^{-}=\left[\phi_{\alpha}, f_{\alpha}\right]_{P}
$$

and it follows that

$$
l_{\alpha \beta} v_{\alpha \beta} f_{\beta}=f_{\alpha} .
$$

As the orbits of $P$ on $G / \bar{P}$ are $P$ stable they define subvarieties of the fibration $R^{+}(G / \bar{P})$ (see Murray (1984)) and, in particular we can consider the set in $\mathscr{T}$ where the section $R^{-}$doesn't intersect the open orbit. It follows from Hitchin (1982) and Murray (1984) that this is a compact algebraic curve $S$. Locally $U_{\alpha} \cap S$ is the curve where $f_{\alpha}$ intersects the complement of the open orbit $V \bar{P}$ in $G / \bar{P}$.

Away from the points of $S \cap U_{\alpha} \cap U_{\beta}$ the $v_{\alpha \beta}$ are determined by Eq. (3.7) because $V$ acts freely on $V \bar{P}$. Because $S$ is a curve, its complement is dense in $U_{\alpha} \cap U_{\beta}$, so that the $v_{\alpha \beta}$ are determined everywhere by knowing the $f_{\alpha}$.

Notice that because $P$ doesn't act freely on $V \bar{P}$ we cannot determine the $p_{\alpha \beta}$ from Eq. (3.7) unless we know the $l_{\alpha \beta}$. This is the reason for introducing the idea of transition sections and the bundle $M$.

The correct interpretation of the $f_{\alpha}$ is also to use the bundle $M$ and to define local sections of $M(G / \bar{P})$ by $\left[\hat{\phi}_{\alpha}, f_{\alpha}\right]$. These do not define a global section as on overlaps $U_{\alpha} \cap U_{\beta}$ they are related by

$$
\left[\hat{\phi}_{\alpha}, f_{\alpha}\right]=\hat{v}_{\alpha \beta}\left[\hat{\phi}_{\beta}, f_{\beta}\right] .
$$

However they do define a global principal part if we generalise the definition of Gravesen to sections of bundles. We have the sheaf $M(\mathscr{M r})$ of "meromorphic sections of $M(V)$ " that is, sections of $M(G / \bar{P})$ intersecting $M(V \bar{P})$. The quotient sheaf $M(\mathscr{M} r) / M(V)=M(\mathscr{P} r)$ is the sheaf of principal parts and the $\left[\hat{\phi}_{\alpha}, f_{\alpha}\right]$ define a global section of this which we call the principal part of the monopole. It can be checked that changing the sections $\phi_{\alpha}$ leaves the principal part unchanged.

It should be noted that these sheaves are sheaves of sets rather than the, perhaps more familiar, sheaves of abelian groups or vector spaces. Definitions can be found in Tennison (1975). In any case it is hoped the local definitions are clear.

It is immediate from the discussion above that a monopole is determined by its principal part.

The reader familiar with sheaf theory will note that this construction of $v_{\alpha \beta}$ from $f_{\alpha}$ resembles the construction of the coboundary of an element in sheaf cohomology. To see how to make sense of this we will make a short one paragraph digression that can be safely ignored if the reader wishes. We need to consider sheaves of pointed sets, that is sheaves whose stalks are sets with a distinguished element (the "point") and exact sequences of sheaves of pointed sets where the 
sequence of pointed sets

$$
A \stackrel{f}{\longrightarrow} B \stackrel{g}{\longrightarrow} C
$$

is called exact at $B$ if $f(A)$ is equal to the preimage under $g$ of the point in $C$. (In the case that $A, B, C$ are groups the point is taken to be the identity and then this becomes the more familiar definition of exactness.) Then we have two exact sequences of sheaves of pointed sets, whose first terms are sheaves of groups;

$$
\begin{aligned}
& 0 \rightarrow R^{+}(V) \rightarrow R^{+}(\mathscr{M} r) \rightarrow R^{+}(\mathscr{P} r)=M(\mathscr{P} r) \rightarrow 0, \\
& 0 \rightarrow M(V) \rightarrow M(\mathscr{M} r) \rightarrow M(\mathscr{P P} r) \rightarrow 0 .
\end{aligned}
$$

The above can then be reinterpreted as saying that the principal part $\mathscr{F}$ of the monopole is the image in $H^{0}(\mathscr{T}, M(\mathscr{P} r))$ of the element $R^{-}$of $H^{0}\left(\mathscr{T}, R^{+}(\mathscr{P} r)\right)$ under the map induced by (3.9). Similarly, the statement that $\mathscr{F}$ determines the monopole is then that $\left[v_{\alpha \beta}\right] \in H^{1}(\mathscr{T}, M(V))$ is the image of $\mathscr{F} \in H^{0}(\mathscr{T}, M(\mathscr{P} r))$ under the coboundary map corresponding to 3.10. This interpretation makes the construction a non-abelian version of the original construction in Hitchin (1982) where the monopole bundle is recovered by applying a coboundary map to the section of $L^{2}$ supported over the spectral data.

Lastly let us define the spectral data of the monopole. If $U_{\alpha}$ does not intersect $S$ then $f_{\alpha}$ is a map into $V$ and therefore in the same coset as the constant map 1 . It is therefore determined! The principal part of the monopole is thus in the sense, supported on $S$ and we shall denote its restriction to $S$ by $f$. This pair $(S, f)$ is the spectral data of the monopole and it is immediate from the discussion above that the spectral data determines the bundle $Q$ without restriction of any kind. (More correctly we should define the spectral data to be $S$ and the orbit of $f$ under the automorphism group of the bundle $M$. Whether or not we do this is connected with whether or not we frame the monopole.)

From the more involved structure theory of $G / \bar{P}$ (see for instance Bernstein, Gel'fand and Gelfand (1973)) we can show as in Murray (1984) that the curve $S$ is a union of $r-d$ curves $S_{i}$ indexed by the elements of $\Delta$ not in $\Delta_{0}$. The degree of $S_{i}$ is twice the magnetic charge $m_{i}$. The curves $\left\{S_{i}\right\}$ we call the spectral curves of the monopole.

To finally complete the result we have to worry about the real structure. We have determined the monopole as a $G$ monopole and we want to know that the original $K$ reduction is also determined. However if there were two different reductions to $K$ compatible with the original connection and Higgs field then this would induce a non-trivial automorphism of the original monopole, and if we assume that the monopole is irreducible this cannot happen (Murray (1984)). So we have proved.

Theorem. The irreducible monopole is determined by its spectral data.

The spectral data as we have defined it here is a rather abstract object. In the next section we shall show that for a general monopole with maximal symmetry breaking our new definition is equivalent to the one in Murray (1984) in terms of curves $S_{i}$ indexed by the Dynkin diagram of the group and a splitting of the intersections $S_{i} \cap S_{j}$ when $i$ and $j$ are joined on the Dynkin diagram. 


\section{The Maximal Symmetry Breaking Case}

We now consider the case of maximal symmetry breaking when $P$ is a Borel subgroup $B$. We review first some of the results from Murray (1984).

Let $Y_{i}$ be the $G$-module with lowest weight the negative of the fundamental weight $\lambda_{i}$ corresponding to the simple root $\alpha_{i}$, and let $Y_{i}(\mu)$ denote the irreducible summand under the action of $T$ with weight $\mu$. Then we have

$$
Y_{i}=Y_{i}\left(-\lambda_{i}\right) \oplus Y_{i}\left(-\lambda_{i}+\alpha_{i}\right) \oplus \cdots,
$$

and we denote by $Z$ the direct sum of all the irreducible summands except the lowest. We then define the following vector bundles on $\mathscr{T}$ :

$$
\begin{aligned}
E_{1}^{-} & =R^{-}\left(Y_{i}\left(-\lambda_{i}\right)\right), \\
E_{2}^{-} & =R^{-}\left(Y_{i}\left(-\lambda_{i}\right) \oplus Y_{i}\left(-\lambda_{i}-\alpha_{i}\right)\right), \\
E_{1}^{+} & =R^{+}(Z), \\
E & =Q\left(Y_{i}\right)=R^{+}\left(Y_{i}\right)=R^{-}\left(Y_{i}\right) .
\end{aligned}
$$

These fit together to form a commuting diagram

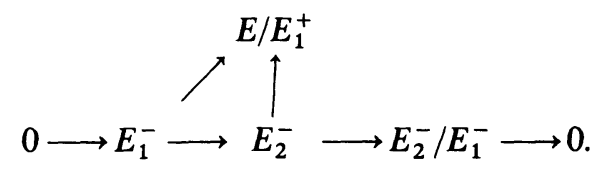

Using the identification in (2.13) this diagram becomes

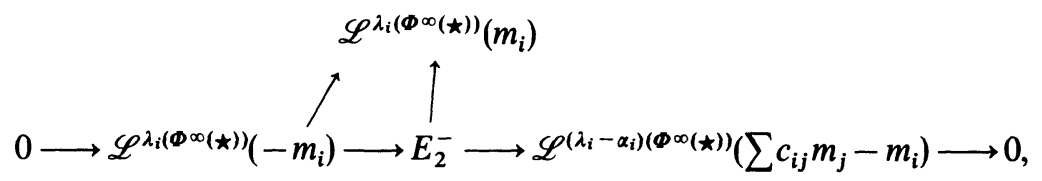

where

$$
c_{i k}=\frac{-\left(\alpha_{k}, \alpha_{i}\right)}{\left(\alpha_{i}, \alpha_{i}\right)}
$$

and (,) is the Killing form. The spectral curve $S_{i}$ is defined by the vanishing of the map

$$
\psi_{i}: E_{1}^{-} \rightarrow E / E_{1}^{+},
$$

and from the commuting diagram (4.3) over $S_{i}$ the middle map factors to define a section

$$
\xi_{i} \in H^{0}\left(S_{i}, \mathscr{L}^{-\alpha_{i}(\Phi \infty(\star))}\left(\sum_{j \neq i} c_{i j} m_{j}\right)\right) .
$$

To relate the $\psi_{i}$ and $\xi_{i}$ to the principal parts we will define them by an equivalent method which begins on $G / \bar{B}$, extends to $R^{+}(G / \bar{B})$ by forming associated bundles and then pulls back to $\mathscr{T}$ by $R^{-}$. We begin this procedure with a discussion about the Schubert cells on $G / \bar{B}$. 
The orbits of $V$ on the manifold $G / \bar{B}$ decompose it into cells

$$
X_{w}=V w \bar{B},
$$

where $w$ runs over the elements of the Weyl group $W=N(T) / T$ or more correctly their coset representatives in the normaliser of the torus $N(T) \subset G$. The closures of these cells are algebraic subvarieties of $G / \bar{B}$. The cell $V e \bar{B}$ is open and there are $r$ cells of codimension one indexed by the simple root reflections $\sigma_{i}=\sigma_{\alpha_{i}}$ in the hyperplanes orthogonal to $\alpha_{i}$ or the nodes $i$ of the Dynkin diagram. If the nodes $i$ and $j$ are joined on the Dynkin diagram then the intersection $\bar{X}_{\sigma_{i}} \cap \bar{X}_{\sigma_{j}}$ is the union of $\bar{X}_{\sigma_{i} \sigma_{j}}$ and $\bar{X}_{\sigma_{j} \sigma_{i}}$. If the nodes $i$ and $j$ are not joined then $\bar{X}_{\sigma_{i}} \cap \bar{X}_{\sigma_{j}}=\bar{X}_{\sigma_{i} \sigma_{j}}=\bar{X}_{\sigma_{j} \sigma_{i}}$.

Defining the following associated vector bundles on $G / \bar{B}$

$$
\begin{aligned}
\mathscr{L}\left(-\lambda_{i}\right) & =G \times_{\bar{B}} Y_{i}\left(-\lambda_{i}\right), \\
\mathscr{L}\left(-\lambda_{i},-\lambda_{i}+\alpha_{i}\right) & =G \times_{\bar{B}}\left(Y_{i}\left(-\lambda_{i}\right) \oplus Y_{i}\left(-\lambda_{i}+\alpha_{i}\right)\right), \\
\mathscr{L}\left(-\lambda_{i}+\alpha_{i}\right) & =G \times_{{ }_{B}}\left(Y_{i}\left(-\lambda_{i}\right) \oplus Y_{i}\left(-\lambda_{i}+\alpha_{i}\right) / Y_{i}\left(-\lambda_{i}\right)\right),
\end{aligned}
$$

we have the diagram

$$
0 \longrightarrow \mathscr{L}\left(-\lambda_{i}\right) \longrightarrow \mathscr{L}\left(-\lambda_{i},-\lambda_{i}+\alpha_{i}\right) \longrightarrow \mathscr{L}\left(-\lambda_{i}+\alpha_{i}\right) \longrightarrow 0 .
$$

The diagonal map vanishes on the $i^{\text {th }}$ Schubert variety $\bar{X}_{i}$ and over it the vertical map factors to give a map

$$
\mathscr{L}\left(-\lambda_{i}+\alpha_{i}\right)_{\mid \bar{X}_{i}} \rightarrow \bar{X}_{i} \times Y_{i} / Z
$$

The group $B$ acts on this diagram and we can form associate fiberings with $R^{+}$. The section $R^{-}$of $R^{+}(G / \bar{B})$ then pulls this back to give the diagram of vector bundles (4.2) or (4.3). Because the orbits are $B$ stable we can consider the associated fiberings

$$
R^{+}\left(X_{w}\right) \subset R^{+}(G / \bar{B})
$$

It is shown in Murray (1984) that using the section $R^{-}$of $R^{+}(G / \bar{B})$

$$
S_{i}=R^{-*} R^{+}\left(\bar{X}_{\sigma_{i}}\right)
$$

and moreover that if we define

$$
S_{i, j}=R^{-*} R^{+}\left(\bar{X}_{\sigma_{i} \sigma_{j}}\right)
$$

so that when $i$ and $j$ are joined on the Dynkin diagram

then the divisor of $\xi_{i}$ is

$$
S_{i} \cap S_{j}=S_{i j} \cup S_{j i}
$$

$$
\sum_{k \neq i} c_{k i} S_{k i}
$$

We shall show that a general, unframed, irreducible monopole, for any choice 
of $K$, is determined by the spectral curves and the division $S_{i} \cap S_{j}=S_{i j} \cup S_{j i}$ when $i$ and $j$ are joined on the Dynkin diagram (that $c_{i j} \neq 0$ ). The proof will consist of a number of steps. First we shall show that the $\psi_{i}$ and $\xi_{i}$ determine the principal part of a framed monopole. Then, we prove that the $\psi_{i}$ are determined by the framing and the curve $S_{i}$, Lastly we note that the $\xi_{i}$ are normalised by the real structure so that there is only the freedom of multiplying each of them by a phase and that this multiplication corresponds to acting by the torus on the framing.

The proof that the $\psi_{i}$ and the $\xi_{i}$ determine the monopole involves looking at local representatives of the principal parts so we will collect first some formulae in local trivialisations.

Choose trivialisations $\phi_{\alpha}^{+}, \phi_{\alpha}^{-}$of $R^{+}, R^{-}$respectively which project to standard trivialisations of $M$ and $\bar{M}$ so that on intersections they satisfy

$$
\begin{aligned}
& \phi_{\alpha}^{+}=\phi_{\beta}^{+} b_{\beta \alpha}^{+}=\phi_{\beta}^{+} l_{\beta \alpha}^{+} v_{\beta \alpha}^{+}, \\
& \phi_{\alpha}^{-}=\phi_{\beta}^{-} b_{\beta \alpha}^{-}=\phi_{\beta}^{-} l_{\beta \alpha}^{-} v_{\beta \alpha}^{-} .
\end{aligned}
$$

Because $R^{+}$and $R^{-}$lie inside $Q$ we can define $F_{\alpha}$ by $\phi_{\alpha}^{+} F_{\alpha}=\phi_{\alpha}^{-}$on each $U_{\alpha}$ and we see that

$$
F_{\beta}=b_{\beta \alpha}^{+} F_{\alpha} b_{\alpha \beta}^{-} .
$$

In the $\phi_{\alpha}^{+}$trivialisation the section $R^{-}$of $Q^{+}(G / \bar{B})=R^{+}(G / \bar{B})$ is

$$
R^{-}=\left[\phi_{\alpha}^{-}, \bar{B}\right]_{G}=\left[\phi_{\alpha}^{+}, F_{\alpha} \bar{B}\right]_{B} .
$$

Let $f_{\alpha}=F_{\alpha} \bar{B}$. Then these are the local representatives for the principal part of the monopole. We want to now relate these to the diagram (4.3) and $\psi_{i}$ and $\xi_{i}$.

Consider first

$$
R^{+}\left(\mathscr{L}\left(-\lambda_{i}\right)\right)=R^{+} \times{ }_{B}\left(G \times{ }_{\bar{B}} Y_{i}\left(-\lambda_{i}\right)\right) .
$$

$R^{-}$determines the section $\left[\phi_{\alpha}^{+}, F_{\alpha} \cdot \bar{B}\right]$ of $R^{+}(G / \bar{B})$ and therefore the fibre of (4.19) over the image of $R^{-}$is

$$
\left[\phi_{\alpha}^{+},\left[F_{\alpha}, Y_{i}\left(-\lambda_{i}\right)\right]_{\bar{B}}\right]_{B} .
$$

Local sections of this are given by $\left[\phi_{\alpha}^{+},\left[F_{\alpha}, c\right]_{\bar{B}}\right]_{B}$, where $c \in Y_{i}\left(-\lambda_{i}\right)$ and as expected over intersections we have

$$
\begin{aligned}
{\left[\phi_{\beta}^{+},\left[F_{\beta}, c\right]_{\bar{B}}\right]_{B} } & =\left[\phi_{\beta}^{+},\left[b_{\beta \alpha}^{+} F_{\alpha} b_{\alpha \beta}^{-}, c\right]_{\bar{B}}\right]_{B} \\
& =\left[\phi_{\beta}^{+} b_{\beta \alpha}^{+},\left[F_{\alpha}, b_{\alpha \beta}^{-} \cdot c\right]_{\bar{B}}\right]_{B} \\
& =\left[\phi_{\alpha}^{+},\left[F_{\alpha},-\lambda_{i}\left(b_{\alpha \beta}^{-}\right) \cdot c\right]_{\bar{B}}\right]_{B} \\
& =-\lambda_{i}\left(l_{\alpha \beta}^{-}\right)\left[\phi_{\alpha}^{+},\left[F_{\alpha}, c\right]_{\bar{B}}\right]_{B} .
\end{aligned}
$$

The bundles of the form $R^{+}(\mu)$, for some character $\mu$, are, because of the framing, canonically isomorphic to $W^{+}(\mu)$ and therefore have canonical local sections $\left[\hat{\phi}_{\alpha}, 1\right]_{L}$. We shall denote all these local sections by $\sigma_{\alpha}$ and similarly for $R^{-}$.

In local co-ordinates the map defining the spectral curve is then

$$
\psi_{i}: \sigma_{\alpha} \mapsto \frac{F_{\alpha} \cdot c+Z}{c+Z} \cdot \sigma_{\alpha} .
$$


Let us explain what we mean by this formulae. Recall from the beginning of Sect. 4 that $Y_{i}$ is the (finite dimensional) representation of lowest weight $-\mu_{i}$ and $Z$ is the sum of all the weight spaces except the lowest so that $Y_{i} / Z$ is a one-dimensional vector space. The vectors $F_{\alpha} \cdot c+Z$ and $c+Z$ are vectors in this one dimensional vector space. The vector $F_{\alpha} \cdot c+Z$ is therefore a complex multiple of the non-zero vector $c+Z$. We denote this complex multiple by

$$
\frac{F_{a} \cdot c+Z}{c+Z}
$$

and note that it is zero when $F_{\alpha} \cdot c$ is in $Z$.

If $d$ is an element of $Y_{i}\left(-\lambda_{i}+\alpha_{i}\right)$ we can define sections of the pullback of

by

$$
\mathscr{L}\left(-\lambda_{i},-\lambda_{i}+\alpha_{i}\right) / \mathscr{L}\left(-\lambda_{i}\right)
$$

$$
\left[\phi_{\alpha}^{+},\left[F_{\alpha}^{+}, d\right]\right]
$$

The $\operatorname{map} \xi_{i}$ is therefore in local co-ordinates

$$
\xi_{i}: \sigma_{\alpha} \mapsto \frac{F_{\alpha} \cdot d+Z}{c+Z} \cdot \sigma_{\alpha}
$$

This map is defined on all of $U_{\alpha}$, but only on $S_{i} \cap U_{\alpha}$ does it transform in such a manner as to be a section of the correct bundle. Next that if we left multiply the $F_{\alpha}$ by maps into $V$ then these definitions are unchanged, and so the $\psi_{i}$ and the $\xi_{i}$ are determined by the principal part of the monopole.

Consider the case of $S L(2, \mathrm{C})$, with $Y=\mathrm{C}^{2}, B$ the subgroup of lower triangular matrices, $\bar{B}$ the subgroup of upper triangular matrices,

and

$$
c=\left(\begin{array}{l}
1 \\
0
\end{array}\right)
$$

Writing $F_{\alpha}$ as

$$
d=\left(\begin{array}{l}
0 \\
1
\end{array}\right) \text {. }
$$

$$
F_{\alpha}=\left(\begin{array}{ll}
w & x \\
y & z
\end{array}\right)
$$

we see that the map defining $S$ is

and $\xi$ is

$$
\sigma_{\alpha} \mapsto w \sigma_{\beta}
$$

$$
\sigma_{\alpha} \mapsto x \sigma_{\alpha} .
$$

Notice that at a point of $S_{i}$ we have $w=0$ and therefore $1=\operatorname{det} F_{\alpha}=-y x$.

We come now to the proof of the first part of our theorem. Assume that we have two collections of representatives $f_{\alpha}, \hat{f}_{\alpha}$ of the principal parts of two monopoles with the same $\psi_{i}$ and $\xi_{i}$. Then it is sufficient to show that these $f_{\alpha}, \hat{f}_{\alpha}$ represent the same principal part, that is there is a collection of maps

$$
v_{\alpha}: U_{\alpha} \rightarrow V
$$


such that

$$
v_{\alpha} f_{\alpha}=\hat{f}_{\alpha} .
$$

Now as the $f_{\alpha}$ and the $\hat{f}_{\alpha}$ have the same spectral curves we know that there are maps

$$
v_{\alpha}: U_{\alpha}-\bigcup_{i} S_{i} \rightarrow V
$$

such that (4.33) holds over $U_{\alpha}-\cup S_{i}$. It suffices to show that these $v_{\alpha}$ extend to all of $U_{\alpha}$, moreover as the monopole is general the intersections of the spectral curves are of codimension 2, so by Hartogs' theorem it suffices to show that the $v_{\alpha}$ extend to $U_{\alpha}$ minus the intersections of the spectral curves and the singular points of the spectral curves. We can work therefore in an open set $U$ as above which intersects only one curve and contains none of its singular points.

In the $S L(2, C)$ case (which of course we know to be true by Hitchin's original results (Hitchin (1982)) we have $F_{\alpha}$ defined as a matrix as in Eq. (4.29) and similarly for $\hat{F}_{\alpha}$ with hats on the matrix entries. Because both these monopoles have the same $\psi$ and the same $\xi$ defined by Eqs. (4.30) and (4.31) we must have that $w=\hat{w}$ over $U_{\alpha}$ and $y=\hat{y}$ over $S \cap U_{\alpha}$. Then from (4.34) we have, away from $S$,

$$
v_{\alpha} F_{a} \bar{B}=\hat{F}_{\alpha} \bar{B} \text {. }
$$

Writing this as

$$
\left(\begin{array}{ll}
1 & 0 \\
\rho & 1
\end{array}\right)\left(\begin{array}{ll}
w & x \\
y & z
\end{array}\right)\left(\begin{array}{l}
1 \\
0
\end{array}\right)=\gamma\left(\begin{array}{ll}
\hat{w} & \hat{x} \\
\hat{y} & \hat{z}
\end{array}\right)\left(\begin{array}{l}
1 \\
0
\end{array}\right)
$$

gives

$$
w=\gamma \hat{w}, \quad \rho=\frac{\gamma \hat{y}-y}{w},
$$

and hence $\gamma=1$. The curve $S \cap U$ is the divisor of $w=0$, which vanishes with multiplicity one, so that $\rho$ and therefore $v$ extends to all of $U$.

Returning to the general case we choose an open set $U \subset U_{\alpha}$ which only intersects $S_{i}$ and denote restriction from $U_{\alpha}$ to $U$ by removing the subscript $\alpha$. If we compose $f=f_{i \mid U}$ with the projection from $G / \bar{B}$ to $G / \bar{P}$, where $\bar{P}$ is the parabolic whose Lie algebra is the sum of the Lie algebra of $\bar{B}$ and the $i^{\text {th }}$ simple root space, then the image lies in the open cell of $G / \bar{P}$. This open cell is an orbit of $\widetilde{V}$, the subgroup of $V$ generated by all the simple root spaces except that corresponding to the $i^{\text {th }}$ simple root. The fibre over $\bar{P}$ of $G / \bar{B} \rightarrow G / \bar{P}$ is $G_{i} / \bar{B} \cong P_{1}(C)$, where $G_{i}$ is the $S L(2, C)$ generated by the $i^{\text {th }}$ simple root and its negative and $\bar{B}_{i}=G_{i} \cap \bar{B}$. Hence there is a map $u: U \rightarrow \widetilde{V}$ such that

$$
u^{-1} f: U \rightarrow G_{i} / \bar{B}_{i} \subset G / \bar{B} .
$$

We have seen above that the $\psi_{i}$ and $\xi_{i}$ depend only on the principal part of the monopole so we can replace $F_{\alpha}$ and $\hat{F}_{\alpha}$ by $u^{-1} F_{\alpha}$ and $\hat{u}^{-1} \hat{F}_{\alpha}$ so that $f$ and $\hat{f}$ take their values in $G_{i} / \bar{B}_{i} \subset G / \bar{B}$. This means that we have $F \bar{B}=g_{i} \bar{B}$ for some $g_{i}$ taking values in $G_{i}$ and similarly with hats so

$$
F=g_{i} \bar{b}, \quad \hat{F}=\hat{g}_{i} \hat{\bar{b}}
$$


where $g_{i}$ and $\hat{g}_{i}$ take their values in $G_{i}$, and $b, \hat{b}$ take their values in $\bar{B}$. The vectors $c$ and $d$ in $Y_{i}$ span a two dimensional subspace which is a representation of $G_{i}$; using this to write $g_{i}$ and $\hat{g}_{i}$ in matrix form as in (4.29) we have

$$
F=\left(\begin{array}{ll}
w & x \\
y & z
\end{array}\right) \bar{b}
$$

and similarly with hats. From Eq. (4.22) we see that $\psi_{i}$ is

$$
\psi_{i}: \sigma_{\alpha} \mapsto \frac{F_{\alpha} \cdot c+Z}{c+Z} \sigma_{\alpha},
$$

so taking into account the action of the Borel $\bar{B}$ this becomes

$$
\psi_{i}: \sigma_{\alpha} \mapsto((-\lambda)(\bar{b})) w \sigma_{\alpha}
$$

over $U$ and similarly for $\hat{F}$ we obtain

$$
\hat{\psi}_{i}: \sigma_{\alpha} \mapsto((-\lambda)(\hat{\bar{b}})) \hat{w} \sigma_{\alpha}
$$

over $U$. As $\psi_{i}=\hat{\psi}_{i}$ we must have

$$
\left(-\lambda_{i}\right)(\bar{b}) w=\left(-\lambda_{i}\right)(\hat{\bar{b}}) \hat{w}
$$

over $U$.

For the section $\xi_{i}$ we have

$$
\xi_{i}: \hat{\sigma}_{\alpha} \mapsto \frac{F_{\alpha} \cdot d+Z}{c+Z} \cdot \hat{\sigma}_{\alpha}
$$

and therefore

$$
\hat{\sigma}_{\alpha} \mapsto\left(\left(-\lambda_{i}-\alpha_{i}\right)(b)\right) x \hat{\sigma}_{\alpha},
$$

and similarly for $\hat{\xi}_{i}$. As $\hat{\xi}_{i}=\xi_{i}$

$$
\left(\left(-\lambda_{i}-\alpha_{i}\right)(b)\right) x=\left(\left(-\lambda_{i}-\alpha_{i}\right)(\hat{b})\right) \hat{x} .
$$

Consider now the $v=v_{\alpha_{i}} \mid U_{\alpha}$ defined on $U-S_{i}$ such that

$$
v F=\hat{F} \text {. }
$$

As we have replaced $F$ and $\hat{F}$ by $u^{-1} F$ and $\hat{u}^{-1} \hat{F}$ the function $v$ now takes its values in $V \cap G_{i}$.

Proceeding as in the $S L(2, \mathrm{C})$ case we have, away from $S_{i}$,

$$
\left(\begin{array}{ll}
1 & 0 \\
\rho & 1
\end{array}\right)\left(\begin{array}{ll}
w & x \\
y & z
\end{array}\right) \bar{b} c=\gamma\left(\begin{array}{ll}
\hat{w} & \hat{x} \\
\hat{y} & \hat{z}
\end{array}\right) \hat{b} c .
$$

As $\bar{B}$ acts by scalars on $c$ we can absorb this into $\gamma$ and we have that $v$ extends over $S_{i}$ if the $\rho$ defined by

$$
w=\gamma \hat{w}, \quad \rho=\frac{\gamma \hat{y}-y}{w}
$$

extends over $S_{i}$. Note that we have $y=x^{-1}$ over $U \cap S_{i}$ and the same with hats. 
Also the $\bar{b}$ and $\hat{b}$ stabilize the span of $c$ and $d$ and act there by determinant 1 so that

and

$$
-\lambda_{i}(\bar{b}) \cdot\left(\left(-\lambda_{i}-\alpha_{i}\right)(\bar{b})\right)=1
$$

$$
-\lambda_{i}(\hat{\bar{b}}) \cdot\left(\left(-\lambda_{i}-\alpha_{i}\right)(\hat{\bar{b}})\right)=1 \text {. }
$$

Moreover from (4.42) and (4.45)

$$
\gamma=\left(-\lambda_{i}(\hat{\bar{b}})\right)\left(-\lambda_{i}(\bar{b})\right)^{-1}
$$

so that using (4.46)

$$
\begin{aligned}
\gamma \hat{y} & =\left(-\lambda_{i}(\hat{\bar{b}})\right)\left(-\lambda_{i}(\bar{b})\right)^{-1} \hat{y} \\
& =-\left(\left(-\lambda_{i}-\alpha_{i}\right)(\bar{b})\right)\left(\left(-\lambda_{i}-\alpha_{i}\right)(\hat{\bar{b}})\right)^{-1} \hat{x}^{-1} \\
& =-x^{-1} \\
& =y .
\end{aligned}
$$

As the monopole is general, $w$ vanishes with multiplicity one on $S_{i}$ and it follows that $v_{\alpha}$ extends and the two monopoles have the same principal parts so by the theorem in Sect. 3 they are the same monopole.

Next we want to consider the $\psi_{i}$. We see from Murray (1984) that in $\mathbf{R}^{3}$ the bundle $E_{1}^{-}$over a line $\gamma$ is the space of solutions of

$$
\nabla_{\gamma} s-i \Phi s=0
$$

which decay like $\exp \left(-\lambda_{i}\left(\Phi^{\infty}(\star)\right)\right) t$ as $t \rightarrow \infty$ along $\gamma$. Here $s$ is a map from $\gamma$ to $Y_{i}$. Moreover the decay conditions for the monopole mean that as we move $\gamma$ away to infinity, keeping it parallel to $\star$ the solutions approach solutions whose asymptotic value (as $t \rightarrow \infty)$ (suitably renormalized) lies in $Y_{i}\left(-\lambda_{i}\right)$. (See Murray (1984) p. 547 and references therein for more details.) A similar description applies to $E / E_{1}^{+}$and we see that as $\gamma$ approaches $\infty$ the map $\psi_{i}$ approaches the projection $Y_{i}\left(-\lambda_{i}\right) \rightarrow Y_{i} / Z$. Of course here we are implicitly trivializing $L^{-\lambda_{i}\left(\Phi_{i}^{\infty}\right)}$ along the fibre of lines parallel to $\star$. So any two $\psi_{i}$ for the same $S_{i}$ are constant multiples of each other with the same asymptotic form in some suitable trivialization, so they must be equal.

Lastly consider the framing. The torus $T$ acts on the framing and therefore on the $\phi_{\alpha}^{+}$and $\phi_{\alpha}^{-}$. If we follow this action through the discussion above we see that it leaves $\psi_{i}$ unchanged (as we would hope!) and acts on the $\xi_{i}$ by a phase. The real structure mapping $W^{+}$to $W^{-}$can be used to define a conjugate linear map

$$
\mathscr{L}^{-\alpha_{i}\left(\Phi^{\infty}(k)\right)}\left(\sum_{k \neq i} c_{i k} m_{k}\right) \rightarrow \mathscr{L}^{\alpha_{i}\left(\Phi^{\infty}(*)\right)}\left(\sum_{k \neq i} c_{i k} m_{k}\right)
$$

covering the action of $\tau$ on $\mathscr{T}$. If we denote by $\xi_{i}^{*}$ the result of applying this map and $\tau$ to $\xi_{i}$, then $\xi_{i} \xi_{i}^{*}$ is a section over $S_{i}$ with divisor $\sum_{k \neq i} c_{i k} S_{i} \cap S_{k}$. This means that $\xi_{i} \xi_{i}^{*}$ is a multiple of $\prod_{k \neq i} g_{k}^{c_{i k}}$ and this multiple is the same for different monopoles. For more details see Hurtubise and Murray (1989). The important thing is that the different possible $\xi_{i}$, given the spectral curves and the $S_{i j}$, are related by phases and correspond to the action of the torus on the framing. 


\section{Spectral Data and Nahm's Equations}

Let $K$ now be a compact connected Lie group, and let $\chi: K \rightarrow S U(N)$ be a representation, with $\chi: G \rightarrow S l(N, \mathbf{C})$ its complexification. This also induces a map $\chi$ on Lie algebras, and we fix

$$
\chi\left(\Phi^{\infty}(\star)\right)=\mu=i \operatorname{diag}\left(\mu_{1}, \mu_{1}, \ldots, \mu_{1}, \mu_{2}, \ldots, \mu_{2}, \ldots, \mu_{k}, \ldots, \mu_{k}\right),
$$

with $\mu_{1}>\mu_{2}>\mu_{3}>\cdots>\mu_{k}$, and $\mu_{j}$ repeated $s_{j}$ times. Let $r_{j}=s_{1}+s_{2}+\cdots+s_{j}$. We will suppose that $\Phi^{\infty}(\star)$ lies in the fundamental Weyl chamber. Now let $\tilde{L}$ be the centraliser of $\mu$ in $S l(N, \mathbf{C}), \widetilde{P}$ be the group generated by $\tilde{L}$ and the upper triangular matrices, $\tilde{\bar{P}}$ be the group generated by $\tilde{L}$ and the lower triangular matrices, $\widetilde{T}$ be the diagonal matrices in $S l(N, \mathbf{C})$. We can suppose that $T$ maps to $\widetilde{T}, P$ to $\widetilde{P}$, etc., and so $G / P$ is mapped to $S l(N, \mathrm{C}) / \widetilde{P}$, etc.

Under $\chi$, the $K$-monopole we were considering becomes an $S U(N)$-monopole. The corresponding $G$-bundle over $\mathscr{T}$ is then an $S l(N, \mathbf{C})$-bundle, and so defines a rank $N$ vector bundle over $\mathscr{T}$. The induced reductions $\widetilde{R}^{+}, \tilde{R}^{-}$to $\bar{P}, \tilde{P}$ define flags of subbundles $E_{r_{1}}^{+} \subset E_{r_{2}}^{+} \subset \cdots \subset E_{N}^{+}=E$ and $E_{N-r_{k}-1}^{-} \subset E_{N-r_{k}-2}^{-} \subset \cdots \subset E_{N-r_{1}}^{-} \subset$ $E_{N}^{-}=E$, with $\operatorname{rank}\left(E_{j}^{ \pm}\right)=j$.

One then has $S U(N)$ spectral curves $\tilde{S}_{r_{j}}, j \in\{1, \ldots, k\}$, defined (set-theoretically) as the set of those points of $\mathscr{T}$ over which $E_{r_{j}}^{+}$and $E_{N-r_{j}}^{-}$are not transversal; the sheaf $E /\left(E_{r_{j}}^{+}+E_{N-r_{j}}^{-}\right)$is supported on $\widetilde{S}_{r_{j}}$.

In Hurtubise and Murray (1989), solutions to Nahm's equations on the intervals $\left(\mu_{j+1}, \mu_{j}\right)$ were defined for each $S U(N)$ monopole. These are matrix-valued functions $T_{i}(z), i=1,2,3, z \in\left(\mu_{j+1}, \mu_{j}\right)$, satisfying

$$
\frac{d T_{i}}{d z}-\sum_{j, k} \varepsilon_{i j k} T_{j} T_{k}=0 .
$$

Invariantly, the $T_{i}(z)$ appear as endomorphisms of $H^{0}\left(\mathscr{T},\left(E /\left(E_{r_{j}}^{+}+E_{N-r_{j}}^{-}\right)\right) \otimes\right.$ $\mathscr{L}^{-z}(-1)$ ), where $\mathscr{L}^{s}(k)$ is the line bundle over $\mathscr{T}$ with transition function $e^{-s \eta / / \zeta} \zeta^{k}$. (In Hurtubise and Murray (1989), only the case of maximal symmetry breaking was considered; it is straightforward, however, to generalise to this case, at least to obtain solutions; what is less easy is deducing their boundary behaviour.)

In the principal bundle formalism that we are working with, $E_{r_{j}}^{+}, E_{N-r_{j}}^{-}$and $E /\left(E_{r_{j}}^{+}+E_{N-r_{j}}^{-}\right)$can be interpreted as follows. Over the flag manifold $\operatorname{Sl}(N, \mathbf{C}) / \tilde{\bar{P}}$, the trivial bundle $V_{N}$ is filtered by trivial bundles $V_{r_{j}}$ chosen so that they are stabilised by $\widetilde{P}$, and also by the tautological bundles $W_{N-r_{j}}$. The quotient $V_{N} /\left(V_{r_{j}}+W_{N-r_{j}}\right)$ is then a sheaf supported over the $j^{\text {th }}$ codimension one Schubert variety in $S l(N, \mathrm{C}) / \tilde{\widetilde{P}}$. Given an $S U(N)$ monopole, one then has vector bundles $\tilde{R}^{+}\left(V_{r_{j}}\right), \tilde{R}^{+}\left(W_{N-r_{j}}\right)$ over $\tilde{R}^{+}(S l(N, C) / \tilde{\bar{P}})$, defined by lifting the action of $\tilde{P}$ on $S l(N, \mathbf{C}) / \tilde{\bar{P}}$ in the standard way to the fibers of $V_{r_{j}}$ and in the trivial way to the fibers of $W_{N-r_{j}}$. One then obtains $E_{r_{j}}^{+}=\tilde{R}^{-*}\left(\tilde{R}^{+}\left(V_{r_{j}}\right)\right), E_{N-r_{j}}^{-}=\tilde{R}^{-*}\left(\tilde{R}^{+}\left(W_{N-r_{j}}\right)\right)$, and $E /\left(E_{r_{j}}^{+}+E_{N-r_{j}}^{-}\right)=\widetilde{R}^{-*}\left(\widetilde{R}_{\tilde{R}^{+}}^{+}\left(V_{N} /\left(V_{r_{j}}+W_{N-r_{j}}\right)\right)\right.$, where $\widetilde{R}^{-}$is considered as a section $\tilde{\boldsymbol{R}}^{-}: \mathscr{T} \rightarrow \tilde{R}^{+}(\operatorname{Sl}(N, \mathbf{C}) / \tilde{\bar{P}})$. Alternatively given $G$ and $\chi$, one can use $\chi$ to pull back $V_{r_{j}}, W_{N-r_{j}}$, and $V_{N} /\left(V_{r_{j}}+W_{N-r_{j}}\right)$ to $G / \bar{P}$, and think of these "standard sheaves" as being supported over $G / \bar{P}$, instead of $S l(N, \mathrm{C}) / \tilde{\bar{P}}$. Given a $K$-monopole, the 
associated sheaves are then obtained by applying $R^{-*}\left(R^{+}()\right)$, e.g. $E /\left(E_{r_{j}}^{+}+E_{N-r_{j}}^{-}\right)=$ $R^{-*}\left(R^{+}\left(V_{N} /\left(V_{r_{j}}+W_{N-r_{j}}\right)\right)\right.$.

i) Spectral Curves. The sheaf $E /\left(E_{r_{j}}^{+}+E_{N-r_{j}}^{-}\right)$is supported over the curves $\tilde{S}_{r_{j}}$; by results of Murray (1984), these are a sum, with multiplicity, of the $K$-spectral curves $S_{v}$. If $\tilde{\lambda}_{r_{j}}$ is the $r_{j}^{\text {th }}$ fundamental weight of $S l(N, \mathrm{C})$, corresponding to the action of $\operatorname{Sl}(N, \mathbf{C})$ on $\wedge^{r_{j}}\left(\mathbf{C}^{\mathrm{N}}\right)$, let the pullback of $\bar{\lambda}_{r_{j}}$ to the torus of $G$ be the sum $\sum n_{r_{j}, v} \lambda_{v}$, where $\lambda_{v}$ are the fundamental weights of $G$. One then has $\tilde{S}_{r_{j}}=\sum n_{r_{j}, v} S_{v}$.

There exists a way of computing the $n_{r_{j}, v}$ in terms of the weight diagram of the representation, which, as we shall see is quite suggestive of the structure of the solutions to Nahm's equations. The weight diagram is the labelled graph whose vertices are weights of the representation, and in which two vertices are joined by an (oriented) edge if their difference is a positive simple root of $G$. The labelling of the edges is by the positive simple roots.

Let us give the edge corresponding to the simple root $\alpha_{v}$, the length $\alpha_{v}\left(\Phi^{\infty}(\star)\right)$. We note that in the case of non-maximal symmetry breaking, some $\alpha_{v}\left(\Phi^{\infty}(\star)\right)$ 's have zero length (see (2.4), and the paragraphs following it). One can project the diagram onto the line, mapping a weight $\omega$ onto $\omega\left(\Phi^{\infty}(\star)\right)$. This map then naturally is length preserving on the edges. (See Fig. 1.)

We can express $\bar{\lambda}_{r_{j}}$ as the sum of the $r_{j}$ "first" weights of the representation, i.e. if $t$ is a point in $\left(\mu_{j+1}, \mu_{j}\right)$,

$$
\bar{\lambda}_{r_{j}}=\sum_{\omega(\Phi)>t} \operatorname{mult}(\omega) \omega,
$$

where mult $(\omega)$ is the multiplicity of the weight $\omega$.

We shall consider for each weight $\omega_{0}$ and each simple root $\alpha_{v}$ the " $\alpha_{v}$-string" through $\omega_{0}$. This is a connected subgraph of the weight diagram, whose vertices are the $\omega_{0}+j \alpha_{v}, j \in \mathbf{Z}$ which are weights. Let $\left\langle\omega, \alpha_{v}\right\rangle=2\left(\omega, \alpha_{v}\right) /\left(\alpha_{v}, \alpha_{v}\right)$ be the form used to define the Cartan matrix. One has (Humphreys (1972), p. 122)

$$
\sum_{n=-\infty}^{\infty} \operatorname{mult}\left(\omega_{0}+n \alpha_{v}\right)\left\langle\omega_{0}+n \alpha_{v}, \alpha_{v}\right\rangle=0 .
$$

We assign to the edge of the string between $\omega_{0}+j \alpha_{v}, \omega_{0}+(j+1) \alpha_{v}$ the number

$$
k_{\omega_{0}+j \alpha_{v}, \omega_{0}+(j+1) \alpha_{v}}=\sum_{n=j+1}^{\infty} \operatorname{mult}\left(\omega_{0}+n \alpha_{v}\right)\left\langle\omega_{0}+n \alpha_{v}, \alpha_{v}\right\rangle .
$$

One can show that these numbers are positive on the edges of the weight diagram, and zero if $\left(\omega_{0}+j \alpha_{v}, \omega_{0}+(j+1) \alpha_{v}\right)$ is not an edge of the weight diagram. One then has, for all $j$ :

$$
\operatorname{mult}\left(\omega_{0}+j \alpha_{v}\right)\left\langle\omega_{0}+j \alpha_{v}, \alpha_{v}\right\rangle=k_{\omega_{0}+(j-1) \alpha_{v}, \omega_{0}+j \alpha_{v}}-k_{\omega_{0}+j \alpha_{v}, \omega_{0}+(j+1) \alpha_{v}} .
$$

As for the fundamental weights $\lambda_{\rho},\left\langle\lambda_{\rho}, \alpha_{v}\right\rangle=\delta_{\rho v},(5.6)$ tells us that

$$
\operatorname{mult}\left(\omega_{0}\right) \omega_{0}=\sum_{v}\left(k_{\omega_{0}-\alpha_{v}, \omega_{0}}-k_{\omega_{0}, \omega_{0}+\alpha_{v}}\right) \lambda_{v} .
$$

As $\left(\omega_{0}+\alpha_{v}\right)\left(\Phi^{\infty}(\star)\right) \geqq \omega_{0}\left(\Phi^{\infty}(\star)\right) \geqq\left(\omega_{0}-\alpha_{v}\right)\left(\Phi^{\infty}(\star)\right)$, an inductive argument then 


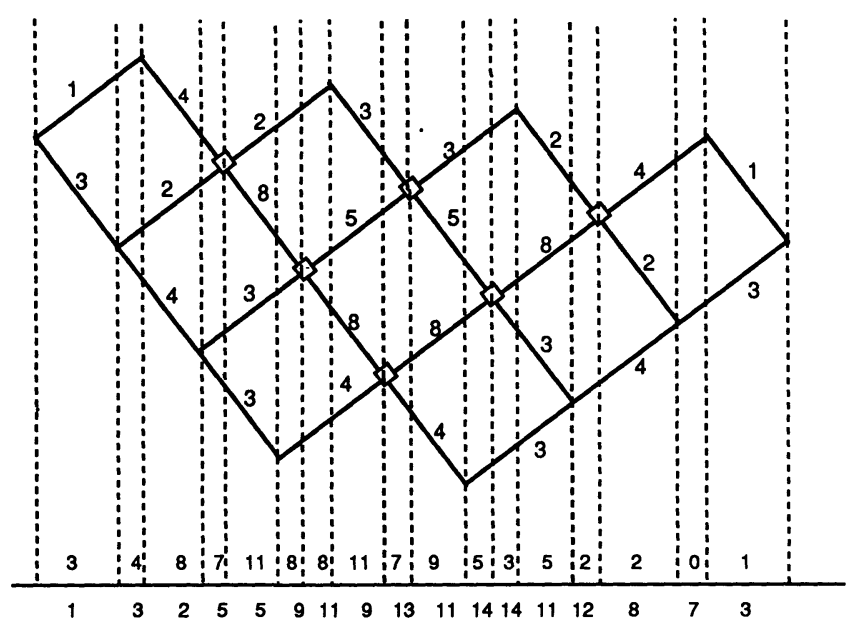

Fig. 1. Weight diagram of the irreducible representation of $S U(3)$ with highest weight $\lambda=\lambda_{1}+3 \lambda_{2}$

Notes on Fig. 1: The segments in the direction $\nwarrow$ are labelled by $\alpha_{1}$, those in the direction $\measuredangle$ are labelled by $\alpha_{2}$. The weights are of multiplicity 1 with the exception of those surrounded by boxes which are of multiplicity 2 . The "lengths" of segments in the diagram are their lengths in the horizontal projection. The numbers on the segments of the diagram are the $k_{\omega_{0}, \omega_{0}+\alpha_{v}}$ 's. The number above the $i^{\text {th }}$ segment from the right on the line is $n_{r, 1}$ and that below is $n_{r, 2}$.

gives

$$
\tilde{\lambda}_{r_{j}}=\sum_{\omega(\Phi)>t} \operatorname{mult}(\omega) \omega=\sum_{v}\left(\sum_{\left\{\omega \mid\left(\omega-\alpha_{v}\right)\left(\Phi^{\infty}(*)\right)<t<\omega\left(\Phi^{\infty}(t)\right)\right\}} k_{\omega-\alpha_{v} \omega}\right) \lambda_{v},
$$

and similarly for the spectral curves, replacing $\tilde{\lambda}$ by $\tilde{S}, \lambda$ by $S$.

Referring to our weight diagram and its projection to the line, the spectral curve $\tilde{S}_{r_{j}}$ corresponding to the flow over $\left(\mu_{j+1}, \mu_{j}\right)$ is a sum of contributions of the segments of the weight diagram lying above that interval.

ii) Nahm's Equations. On the interval $\left(\mu_{j+1}, \mu_{j}\right)$ a solution to Nahm's equation (a "flow") is defined as a section of the endomorphism bundle of a bundle whose fiber at $t$ is the space of sections of $Q_{r_{j}, t}=\left(E /\left(E_{r_{j}}^{+}+E_{N-r_{j}}^{-}\right) \otimes L^{-t}(-1)\right.$. This sheaf is supported over $\tilde{S}_{r_{j}}=\sum_{v} n_{r_{j}, v} S_{v}$; the dimension of its space of sections is $\sum_{v} n_{r_{j}, v} m_{v}$, where $m_{v}$ is the magnetic charge corresponding to $\lambda_{v}$. Considering the above computation of the $n_{r j}, v$, it seems reasonable to conjecture that the flow is actually a "pushdown" of some sort of flow defined over the weight diagram.

In support of this conjecture, we note that in the general $S U(N)$ case, the existence of sections of $\mathscr{L}^{-\alpha_{v}\left(\Phi^{\infty}(\star)\right)}\left(\left(2 \lambda_{v}-\alpha_{v}\right)\left(2 * F^{\infty}(\star)\right)\right)$ over the curve $S_{v}$ was crucial in showing that the appropriate boundary conditions were satisfied on intervals of length $\alpha_{\nu}\left(\Phi^{\infty}(\star)\right)=\left(\mu_{v+1}, \mu_{v}\right)$. In this more general case, one again has such sections, but here the intervals $\left(\mu_{i+1}, \mu_{i}\right)$ are not of these lengths; on the other hand, the edges of the diagram associated to $\alpha_{v}$ are of length $\alpha_{v}\left(\Phi^{\infty}(\star)\right)$. 
An examination of the simplest non-trivial case, however, that of $S O(2 n)$ and its standard representation, suggests that the "pushdown" is nothing so trivial as a direct sum. In this case, studied in Hurtubise and Murray (1989), one has a parallelogram $A B C D$ in the weight diagram, with $A B, C D$ indexed by $\alpha_{-}, A D, B C$ indexed by $\alpha_{+}$. (The corresponding weights $\lambda_{+}, \lambda_{-}$are the highest weights of the spin representations.) On the line, $C, D, B, A$ project to the line in this order to $-\mu_{n-1},-\mu_{n}, \mu_{n}, \mu_{n-1}$. Over the intervals $\left(-\mu_{n-1},-\mu_{n}\right),\left(\mu_{n}, \mu_{n-1}\right)$, the spectral curve is $S_{+}+S_{-}$; over $\left(-\mu_{n}, \mu_{n}\right)$, it is $2 S_{+}$. For general monopoles, there is an isomorphism $\alpha: \mathscr{L}^{\mu_{n}} \rightarrow \mathscr{L}^{-\mu_{n}}$ over $S_{+} \cap S_{-}$. If one sets

$$
\begin{aligned}
V_{t}^{ \pm} & =H^{0}\left(S_{ \pm}, \mathscr{L}^{\mu_{n}-t}\left(m_{+}+m_{-}-1\right)\right), \\
W_{t}^{ \pm} & =H^{0}\left(S_{ \pm}, \mathscr{L}^{-\mu_{n}-t}\left(m_{+}+m_{-}-1\right)\right),
\end{aligned}
$$

associating $V_{t}^{+}$to $C B, V_{t}^{-}$to $B A, W_{t}^{+}$to $A D, W_{t}^{-}$to $C D$, we find:

$$
\begin{aligned}
H^{0}\left(\mathscr{T}, Q_{n-1, t}\right) & =\left\{(v, w) \in V_{t}^{-} \times W_{t}^{+} \mid \alpha(v)=w \text { over } S_{+} \cap S_{-}\right\}, \\
H^{0}\left(\mathscr{T}, Q_{n, t}\right) & =\left\{(v, w) \in V_{t}^{+} \times W_{t}^{+} \mid \alpha(v)=w \text { over } S_{+} \cap S_{-}\right\}, \\
H^{0}\left(\mathscr{T}, Q_{n-1, t}\right) & =\left\{(v, w) \in V_{t}^{+} \times W_{t}^{-} \mid \alpha(v)=w \text { over } S_{+} \cap S_{-}\right\} .
\end{aligned}
$$

Thus the relevant bundles on the line are not products of bundles defined over each edge of the diagram over that portion of the line, but rather subbundles of such products defined by constraints associated to the intersections of the curves. This should be typical of the general situation; after all, sections of $Q_{r_{j}, t}$ over a union of curves can be thought of as sections of $Q_{r_{j}, t}$ over each curve, constrained by equality at the intersections.

Acknowledgements. We would like to thank M. Zabcic for useful conversations and the referee for useful comments. We would also like to thank the Institute for Advanced Study, Princeton and the School of Mathematical Sciences, Institute of Advanced Studies, Canberra for their hospitality and support.

\section{References}

Bernstein, I. N., Gel'fand, I. M., Gel'fand, S. I.: Schubert cells and the cohomology of the spaces G/P. Russ. Math. Surv. 28(3), 1-26 (1973)

Garland, H., Murray, M. K.: Why instantons are monopoles. Commun. Math.Phys. 121, 85-90 (1989)

Gravesen, J.: On the topology of spaces of holomorphic maps. D.Phil Thesis. Oxford. (1987)

Grothendieck, A.: Sur la classification des fibrés holomorphes sur la sphère de Riemann. Am. J. Math. 79, 121-138 (1957)

Hitchin, N. J.: Monopoles and geodesics. Commun. Math. Phys. 83, 579-602 (1982)

Hitchin, N. J.: On the construction of monopoles. Commun. Math. Phys. 89, 145-190 (1983)

Hitchin, N. J.: Murray, M. K.: Spectral curves and the ADHM construction. Commun. Math. Phys. 114, 463-474 (1988)

Humphreys, J. E.: Introduction to Lie algebras and representation theory. Berlin, Heidelberg, New York: Springer 1972

Humphreys, J. E.: Linear Algebraic Groups. Berlin, Heidelberg, New York: Springer 1981

Hurtubise, J. C., Murray, M. K.: On the construction of monopoles for the classical groups. Commun. Math. Phys. 122, 35-89 (1989)

Kobayashi, I. N., Nomizu, K.: Foundations of differential geometry, Vol. 1. New York: Interscience, Wiley 1969 
Murray, M. K.: Non-abelian magnetic monopoles. Commun. Math. Phys. 96, 539-565 (1984)

Murray, M. K.: Stratifying monopoles and rational maps. Commun. Math. Phys. 125, 661-674 (1989)

Nahm, W.: Self-dual monopoles and calorons. In: Group Theoretical Methods in Physics. Denado, G. et al. (eds), Trieste. Lecture Notes in Physics vol. 201. Berlin, Heidelberg, New York: Springer 1983 Pressley, A., Segal, G.: Loop groups. Oxford: Oxford University Press 1986

Tennison, B. R.: Sheaf Theory. Lond. Math. Soc. Lecture Note Series 20. London, New York, Melbourne: Cambridge University Press 1975

Communicated by A. Jaffe 